\title{
Changing Paradigm of Breast Cancer
}

\author{
K. S. Gopinath
}

Published online: 20 October 2011

(C) Indian Association of Surgical Oncology 2011

[1] Cancer of the Breast has emerged as a leading site of cancer in most urban population of India. It is estimated that 90,000 new cases of breast cancer are detected annually. National cancer registry programme has shown a statistical rising trend in the incidence rate of Breast cancer. In the hospital cancer registries Breast cancer constitutes $14.32 \%$ to $30 \%$ of all cancers in women [2]. The variation noticed after development of Atlas of cancer in India, showed higher microscopic incidence of breast cancer in peripheral parts of India [3].

The debate continues regarding screening programme of breast cancer in India how feasible \& what methodology one should adopt in implementing the different programme.

The articles related to problem of breast cancer and its variation has been published in this issue. There is cultural variation in women in different parts of India. The incidence of receptor status varies from western to developing world. This has been published by Suraj et al. on Estrogen receptor negative breast cancer in India do we have higher burden of this type. This clearly depicts their higher triple negative cancer and the facilities available for evaluating these patients for standard of care treatment.

Inamdar et al. publishing the Correlation Between Obesity and High Density Lipoprotein Cholesterol (HDL-C) in Breast Cancer Patients of Southern Rajasthan, a variant observed in that population which leads to future thinking on preventive surgery for cancer in relation to obesity and breast cancer.

\footnotetext{
K. S. Gopinath $(\bowtie)$

Bangalore Institute of Oncology, Ambuja Health Care,

Bangalore 560027, India

e-mail: gopiijso@gmail.com
}

Maji et al.- Onco plastic surgery- our experience in the breast clinic, from Eastern part of the country. Indicating that breast conservative surgery with or without reconstruction is a demand by the patients one has to learn the newer techniques to give a quality of life for the women suffering of Breast cancer.

This issue also highlights complications of axillary dissection both after mastectomy and BCS, by Brar et al. To complete Tripuraneni et al. as given a overview on Radiation therapy in early stage invasive breast cancer. Sameer Damle et al. bringing out the issue of Prophylatic mastectomy in Contralateral Breast cancer.

The other articles in this issue a review by Raghunandan et al on Imaging in staging and Management of Pancreatic ductal adeno carcinomas, and Chamberlain and his colleagues have reviewed the significance of CA 19-A as Bio marker in Pancreatic cancer.

The simple and a day to day problem of FNAC of Thyroid Nodules an article on inadequate cytology in thyroid nodules: should we repeat Aspiration or Follow-Up? By Yoon et al. has extend a curiosity and thinking on this subject what to do.

The breast cancer remains a challenge and changing paradigm has been observed in various aspects of Breast cancer in Indian subcontinent.

\section{References}

1. Nanda Kumar IJSO 2010; Vol.1. 1 8-8 \& 9 The magnitude of cancer breast in India: a summary

2. Consolidated report of Hospital Based cancer registries 2001-2003 National cancer registry Programme (ICMR), Bangalore, 2007

3. Nanda Kumar A, Gupta PC, Gangadharan P, Visweswara RN, Parkin DM (2005) Geography re-visited development of atlas of cancer in India. Int J Cancer 116(5):740-754 